\title{
Research on Evaluation Method of Ecological Environment Quality Based on the Improvement of Human Settlement Environment
}

\author{
Chen $\mathrm{Xi}^{1}$, Gao Xia ${ }^{2, *}$, Li Xianyue ${ }^{1}$ \\ ${ }^{1}$ School of Social Development and Management, Hunan Women's University, Changsha, Hunan, China \\ ${ }^{2}$ Institute of Economic Geography, Hunan University of Finance and Economics, Changsha, Hunan, China
}

\begin{abstract}
Against the background that ecological environment quality has become a hot research topic in recent years, ecological environment quality assessment has emerged as an emerging discipline. By adopting scientific methods, it objectively and quantitatively reveals the real situation of ecological environment quality, and is committed to providing support basis for environmental protection and comprehensive prevention and control. Therefore, how to develop a reasonable eco-environmental quality assessment system has become the focus of attention. Based on this, this paper summarizes the research results in this field at home and abroad, and discusses and illustrates the main existing evaluation methods and evaluation models through horizontal and vertical analysis. The development status of ecological environment quality evaluation research field and the application characteristics of different evaluation methods are analyzed, some problems in theoretical innovation, model construction and application of conclusions are discussed, and the development trend of ecological environment quality evaluation research is proposed based on this.
\end{abstract}

\section{Introduction}

The development of human society has entered the postindustrial era, and all its production activities both depend on the ecological environment and affect the development and changes of the ecological environment. While seeking new socio-economic growth points, we are constantly subjected to the test of environment. How to come out of a sustainable development path under the constraints of ecological environment is the focus of scholars at home and abroad at this stage. Therefore, the understanding and evaluation of ecological environment science will be a necessary link to promote the research in the field of resources and environment, and at the same time will help the construction of ecological civilization and ecological economic development.

In response to such problems, human beings need to understand the quality structure, damage mechanism and governance system of ecological environment more deeply, thus it is important to carry out research on ecological environment quality evaluation in a global scale. Based on recent advances in spatial data information acquisition, processing and analysis techniques and methods such as remote sensing and GIS, ecological environment quality evaluation techniques and methods have transitioned from the initial single-factor survey to comprehensive multi-factor evaluation supported by multi-source data, and the evaluation content has developed from purely natural environment to the direction of integrating natural and social environment. In the process of continuous evolution and development of ecological environment quality evalu-ation methods, it has greatly promoted the generation of comprehensive evaluation methods and realized the transformation from thematic research to comprehensive research and from single method to method integration, which makes the research continue to push forward[1]. Although many experts and scholars at home and abroad have conducted very extensive and fruitful researches on ecological environment quality evaluation, an evaluation paradigm has not yet been formed. Therefore, this paper analyzes the current situation of the research as well as the existing problems on the basis of further synthesis of this research at home and abroad, and introduces the value-added evaluation methods led by environmental value-added related theories, etc., in order to promote the research progress of ecological environmental quality evaluation and other related topics.

\section{Comprehensive evaluation of ecolog- ical environment quality}

Evaluation is actually a process of recognition with regular characteristics in society and is an important basis for decision-making. The evaluation of ecological environment quality is a qualitative or quantitative analysis and prediction of the regional environmental quality based on the data and indicators collected from environmental testing, which is essentially an evaluation of the various elements of the ecological environment 
using different methods, and finally a comprehensive and objective evaluation of the environmental quality of the region. Among them: qualitative evaluation generally selects the indicators that have a greater impact on the ecological environment, and evaluates the ecological environment according to the size or the degree of merit of the indicators; while quantitative evaluation adopts a certain formula or model to calculate the indicator system, and evaluates the ecological environment acco-rding to the size of the calculation results.

The environment is a basic element of human existence, closely related to human life and work, and has an important impact on the sustainable development of society. As environmental pollution has become an important issue to be solved, an objective and reasonable evaluation of the current state of the environment is necessary to propose an implementable environmental management strategy, because it is the basis for all other environmental work and is highly relevant to the rest of the work system, and only if this basic work is carried out successfully can we ensure the solid promotion of all subsequent work. It is only when this basic work is carried out successfully that the subsequent work can be firmly promoted[2]. The assessment of environmental conditions not only provides a better understanding of the current environmental quality, identifies problems in the process of environmental management, and provides a better reference for modern environmental protection, but also allows us to predict the factors affecting the environment in social activities, thus reducing the negative impact of social activities on the natural environment and creating a more livable and pleasant living environment for human beings[3].

\section{Analysis of comprehensive environ- mental evaluation methods}

In the evaluation of ecological environment quality, the evaluation method is of great significance as an essential tool, and the single evaluation method usually used cannot ensure the reliability of its results. Therefore, comprehensive evaluation methods are taking center stage, which can enhance the objectivity and rationality of environmental evaluation and provide an important basis for environmental protection and management to meet the actual needs of current environmental protec-tion work. At present, the most widely used ecological environment quality evaluation methods are mainly the following six.

\subsection{Hierarchical analysis method}

Hierarchical analysis was proposed by T.L. Saaty, an American operations researcher, in the early 1970s. The so-called hierarchical analysis method is to stratify the different elements in the environmental evaluation system, and use the requirements of the previous level as a benchmark to systematically standardize this level, then compare the two levels and clarify the weighting of each element, and then through the steps of creating the instruction system and normalization, finally achieve the purpose of calculating the comprehensive evaluation value.

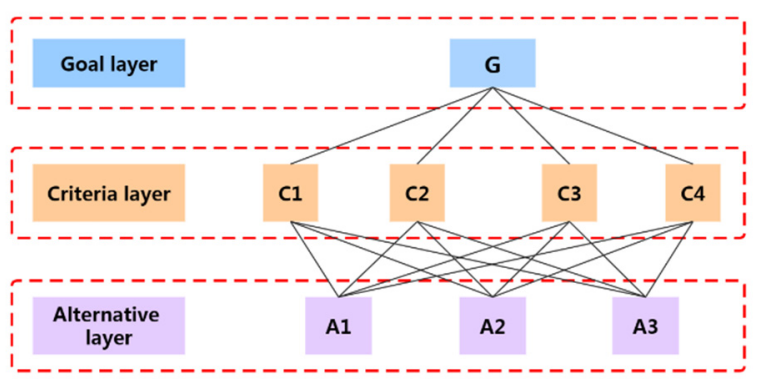

Fig. 1.Hierarchical Analysis Structure Diagram

\subsection{Principal component analysis method}

In the process of comprehensive evaluation of ecological and environmental quality with multiple indicators, the evaluation results of multiple indicators are usually integrated together using a weighting method to obtain a holistic evaluation value. Principal component analysis is a method to simplify the complex relationships among the interrelated variables, and it tries to make the best data simplification for this multivariate integrated crosssection while ensuring the least data missing. In other words, the principle of preserving data integrity is to spatially downscale the higher dimensional variables and study a few linear combinations in the indicator system, while the composite indicators composed of these linear combinations will retain as many of their pre-variance characteristics as possible. This method avoids the interference of human subjectivity and has a strong mathematical theoretical basis. Using this method, numerous scholars have conducted comprehensive evaluations of ecological environment quality in different regions.

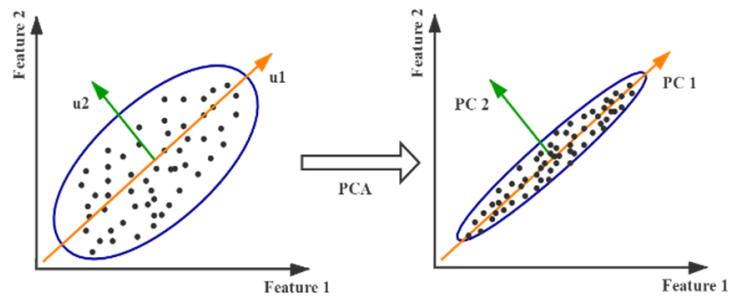

Fig. 2. Model diagram of principal component analysis method

\subsection{Fuzzy comprehensive evaluation method}

Precision had long been the standard of application in the mathematical realm until 1965, when Zadeh first introduced the phenomenon of fuzziness into the scope of mathematical applications, arguing that the human brain has fuzzy characteristics for judging and identif-ying problems. This idea greatly broadened the scope of application of conventional mathematics, and with it, fuzzy mathematics was formally accepted by the scientific community and applied in practice. Based on the orientation of fuzzy mathematical principles and the need 
of realistic evaluation system, the fuzzy compreh-ensive evaluation method came into being. The core theory of this method is to adopt a unique fuzzy transformation approach to objectively and comprehen-sively evaluate complex systems with many influencing factors in a fuzzy environment. The essence of the method is to establish a matrix of index affiliation sets based on the data obtained from environmental monito-ring, and then obtain the final comprehensive evaluation by multiplying the weight sets of each index with the matrix. Under the concept of this method, the ecological environment system is abstracted as a complex complex body influenced by many factors. Therefore, it can better solve the problems with ambiguity and non-determinism.

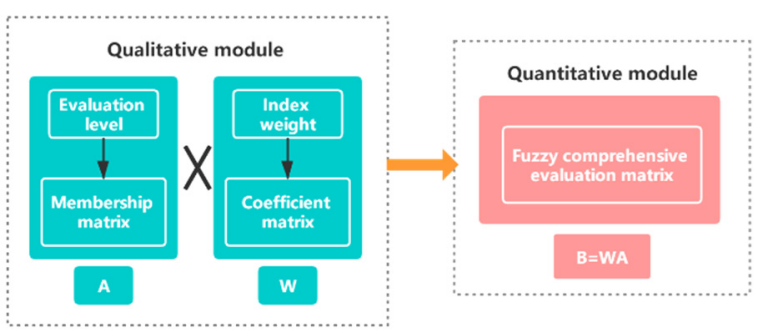

Fig. 3. Fuzzy integrated evaluation method concept map

\subsection{Gray evaluation method}

The first paper "Gray Control System" published by our scholar Professor Deng Julong in 1982 marked the birth of the discipline of gray system. Deng Julong believed that the environmental system essentially belongs to a gray system and can be evaluated in a comprehensive way with the help of gray system theory. The gray evaluation method includes gray cluster analysis and gray correlation analysis: (1) The core of gray cluster analysis is to create whitening functions similar to the affiliation function, so as to achieve the purpose of clustering operation, while the main principle of gray clustering operation is to effectively integrate gray theory into the cluster analysis, by analyzing the relationship between objects far and near, and finally using multivariate analysis to carry out judgment. (2) Gray correlation analysis is a branch of gray system theory, which is in essence a comparative analysis of geometric shapes among several curves. It is believed that the more similar the geometric shapes are, the closer the developmental dynamics are, and the greater the degree of their correlation is, and vice versa, the smaller the degree of correlation is. Although the judgment of geometry based on gray correlation analysis is intuitive, it cannot be quantified, and if there are several curves with similar shapes, it is difficult to judge the degree of correlation of each curve by a direct method.

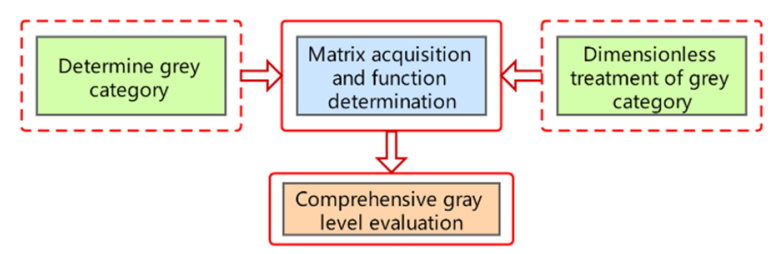

Fig. 4. Flow chart of grey evaluation method

\subsection{BP Artificial Neural Network Evaluation Method}

Artificial neural network (ANN), also known as neurallike network, is an information processing system that can mimic biological neural network, i.e., a complex network system that uses a large number of basic neurons widely connected in series to simulate the thinking pattern of human brain. The system is generally composed of an input layer, an output layer, and several implicit layers, and each neuron in the system interacts with other neurons in the vicinity for information reception and output collection to facilitate the comple-tion of the information processing work of the whole system.BP artificial neural network is a multilayer neural network with forwardlooking characteristics, and it is by far the most widely used type of ANN network type, and the most essential part of its theory is that the adjustment of weights takes the learning algorithm of forward and backward propagation.

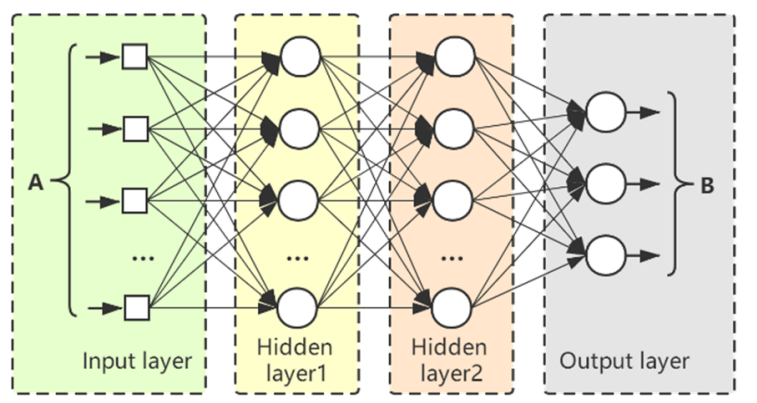

Fig. 5. BP artificial neural network evaluation method structure diagram

\subsection{Object meta-analysis evaluation method}

Since the single factor evaluation results of ecological environment quality are often incompatible with each other, how to make good use of the characteristics of the interaction of correlation functions is the key to evaluate and identify the ecological environment quality belonging to a certain evaluation system level set. The specific steps are: firstly, the classical domain matrix is created with each level of environmental quality standard as a reference, and then the nodal domain matrix is created according to various impact factors, and then the correlation functions of different environmental quality standards corresponding to each numerical criterion are created, and finally, the resulting function values are used to determine the environmental quality level. Finally, the environmental quality levels are determined according to the resulting function values.

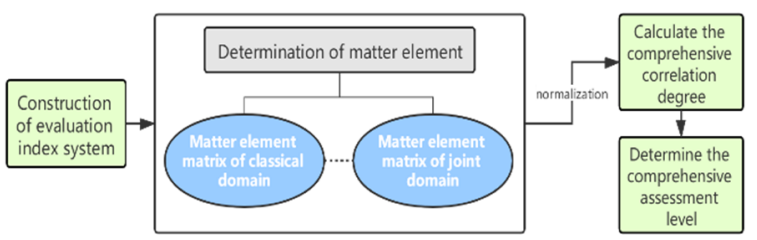

Fig. 6. Flowchart of Object Element Analysis Method 


\section{Analysis of existing problems}

Based on the social development and taking the current situation of ecological and environmental quality as the entry point, discussing the driving mechanism, evolution process and development trend of the evaluation system to serve the sustainable use of resources and socio-economic development is the usual path of ecological and environmental quality evaluation research. This paper lists and discusses the common evaluation methods of ecological environmental quality, and it is clear from the analysis of literature that the existing evaluation method system still has some problems and shortcomings, and the following four aspects are specified[4].

- nsufficient innovation: At this stage, the research on ecological environmental quality evaluation mainly focuses on the methodological level, and the methodological research system is not innovative enough, which is usually based on assessment and multivariate statistical analysis, and the evaluation elements of spatial dynamics and future trend prediction are extremely lacking, so it leads to the increasing contradiction of unclear positioning of hierarchy and evaluation objectives in the research process of ecological environmental quality evaluation system.

- Lack of uniformity: the non-uniformity of the analysis standards of socio-economic indicators, natural condi-tions and other factors inevitably leads to the ecological environment quality evaluation being affected by external interference and maneuvering factors such as incomplete data in the selection of indicators and model selection. Different scholars often have different evaluation index systems and different calculation and analy-sis models in their studies, and a comprehensive and complete ecological environment quality evaluation system involving various objects and scales has not yet been formed, which results in the one-sided and unsystematic description of the evaluation results on the current situation of ecological environment quality, and the evaluation results are not comparable between different evaluation regions[5].

- Strong subjectivity: Due to the imperfect analysis model and the different experts' and scholars' perceptions on the classification and threshold of ecological environment quality evaluation, the determination of index weights, the assignment of scores and the classification of evaluation levels in the process of ecological environment quality evaluation are highly subjective, therefore, the evaluation results do not have direct reference.

- The application research of evaluation results needs to be expanded: the output results of ecological environment quality evaluation research at this stage are only some simple simulation data, but it is still necessary to analyze the results dynamically and then effectively connect with regional ecological environmental protection measures, so as to better serve the zoning management and effective control research of ecological environment, and improve the scientific, effective and practical nature of ecological environment quality research.

\section{Conclusion}

According to the above elaboration, it can be seen that ecological environment quality evaluation is still in the development stage, so we should adhere to the principle of modernization on the basis of the improvement of the previous single evaluation method, supported by a multidisciplinary knowledge system, using the latest technology, coordinating all aspects of the relationship, and adopting a more scientific and comprehensive evaluation method to improve the quality and efficiency of the evaluation work[6]. In practice, we should give full play to the adva6tages of the application of ecological environment quality evaluation and ensure that the evaluation method is continuously optimized to achieve the purpose of more scientific and effective evaluation of the environment, and thus promote the sustainable development of environmental protection.

\section{Acknowledgments}

This work was supported by the scientific research project of Hunan Provincial Education Department in 2020, "Research on Rural Ecological Recreation and Tourism under the Strategy of Rural Revitalization" (No. 20A264).

\section{References}

1. Yang D, Shen S, Zhao D. (2015) Study on vulnerability assessment of ecological environment in Shaanxi Province. Journal of Xi'an University of architecture and Technology (SOCIAL SCIENCE EDITION), 34: 75-80.

2. Zhu L, Cha L. (2009) Research progress of urban ecological environment quality evaluation. Journal of Hubei University of Economics (HUMANITIES AND SOCIAL SCIENCES EDITION), 6: 36-38.

3. Ye C. (2019) Analysis on the application of comprehensive assessment method in Environ-mental Assessment. Environment and develop-ment, 31: 1213.

4. Zhang L. (1994) Application of principal component analysis in Environmental Assessment. Environmental protection, 3: 37-38.

5. Feng C, Li Y. (2008) Application of comprehensive assessment method in Environmental Assessment. Journal of safety and environment, 5: 112-115.

6. Zhai Y. (2008) Research and construction of evaluation index system and database platform of ecological environment quality in Jilin Province [D]. Jilin Agricultural University. 